\title{
IL-10 expression by primary tumor cells correlates with melanoma progression from radial to vertical growth phase and development of metastatic competence
}

Eijun Itakura ${ }^{1}$, Rong-Rong Huang ${ }^{1}$, Duan-Ren Wen ${ }^{1}$, Eberhard Paul ${ }^{2}$, Peter H Wünsch ${ }^{3}$ and Alistair J Cochran ${ }^{1,4,5}$

${ }^{1}$ Department of Pathology and Laboratory Medicine, David Geffen School of Medicine at UCLA, Los Angeles, CA, USA; ${ }^{2}$ Department of Dermatology, Klinikum Nürnberg Nord, Nürnberg, Germany; ${ }^{3}$ Department of Pathology, Klinikum Nürnberg Nord, Nürnberg, Germany; ${ }^{4}$ Department of Surgery, David Geffen School of Medicine at UCLA, Los Angeles, CA, USA and ${ }^{5} U C L A$ 's Jonsson Comprehensive Cancer Center, Los Angeles, CA, USA

Downregulation of the immune system facilitates tumor progression at different stages of cutaneous melanoma. Sentinel nodes, the first lymph nodes on lymphatics draining directly from a primary melanoma, are immune downregulated by tumor-generated immunosuppressive cytokines, including interleukin-10 (IL-10). To better understand the kinetics of sentinel node suppression, we investigated IL-10 expression by melanoma cells and tumor-associated macrophages and lymphocytes at different stages of primary melanoma evolution. We used reverse-transcriptase in situ PCR to identify the cellular sources of IL-10 mRNA in 39 melanomas. IL-10 mRNA was identified in tumor cells of 2 of 6 melanomas in situ (33\%), of 17 of 21 invasive melanomas (81\%) and of 11 of 12 metastatic melanomas (92\%). Higher IL-10 expression correlates with tumor progression, with differences between melanoma in situ, invasive melanoma and metastatic melanoma. In primary melanomas, the IL-10 mRNA content of tumor cells correlates with Clark's level. There was significantly more IL-10 mRNA in vertical growth-phase melanoma cells than in radial growth-phase cells. In a logistic regression model, moderate-to-high IL-10 mRNA expression by tumor cells was significantly associated with vertical growth-phase melanoma. IL-10 mRNA was detected in melanoma-associated macrophages and lymphocytes. In invasive melanomas, IL-10 mRNA reactivity of macrophages decreased as Clark's level increased. Alterations of immunity by IL-10 derived from melanoma cells and melanoma-associated macrophages and lymphocytes potentially facilitate evolution of the primary melanoma and render regional lymph nodes susceptible to metastases.

Modern Pathology (2011) 24, 801-809; doi:10.1038/modpathol.2011.5; published online 11 February 2011

Keywords: cytokines; immune suppression; interleukin-10; melanoma; metastasis; tumor-associated macrophages/lymphocytes

Immune suppression is closely related to the development of lymphatic metastases in patients with melanoma. This is indicated by a reduction in the frequency of paracortical dendritic cells,

Correspondence: Dr E Itakura, MD, PhD, Department of Pathology and Laboratory Medicine, David Geffen School of Medicine at UCLA, 10833 Le Conte Avenue, Room CHS 1P-162, Los Angeles, CA 90095-1732, USA.

E-mail: itakura@ucla.edu

Received 25 July 2010; revised 10 November 2010; accepted 15

November 2010; published online 11 February 2011 activated $\mathrm{T}$ cells and activated endothelia in high endothelial venules in lymph nodes regional to melanoma. ${ }^{1-4}$ The sentinel node, which is the first lymph node to directly receive lymph from a primary melanoma and thus the node most influenced by the primary tumor, is immune suppressed even before nodal metastases are detectable. ${ }^{3}$ In the absence of tumor cells in the sentinel node, immune modulation is likely to be mediated by soluble immune-suppressive factors released by melanoma cells and leukocytes at the primary tumor site. ${ }^{4}$ Interleukin-10 (IL-10) is a prime candidate for this 
role. Several studies have demonstrated elevated levels of IL-10 in the serum obtained from patients with later stages of melanoma. ${ }^{5-7}$ This T-helper type 2 (Th2) cytokine impedes immune functions, including T-cell proliferation, Th1 cytokine production, antigen presentation and lymphokine-activated killer cell cytotoxicity. IL-10 is secreted by melanoma cells, including metastatic melanoma cells ${ }^{8-10}$ as in other cancers and tumor-infiltrating lymphocytes, and has been reported to reduce immune surveillance in patients with advanced melanomas. ${ }^{11}$ Monocytes/macrophages, including tumor-associated macrophages, are significant components of tumor-associated inflammatory infiltrates, and having modulatory activities against the immune system can potentiate tumor progression. ${ }^{12}$ There is a need for further analysis of the tumor microenvironment and the extent that it is orchestrated by bioactive molecules from melanoma cells and their associated inflammatory cells.

Detection of gene expression at the mRNA level is critical for identification of cells that synthesize soluble and secretable proteins, such as cytokines. Immunohistochemistry can detect such proteins in cells that passively acquire them by endocytosis and in cells that synthesize them. The coexistence of specific mRNA and protein delineates cells that synthesize particular proteins. ${ }^{13}$ As described previously, the reverse-transcriptase in situ PCR (RT in situ PCR) technique amplifies small amounts of specific mRNA sequences within cells. ${ }^{14-17}$ In this study, we evaluated the expression of the gene for IL-10 (IL10) in surgically resected melanomas in situ, invasive primary melanomas, metastatic melanomas and melanocytic nevi using the RT in situ PCR technique. This report is part of a series of studies of immunosuppressive networks in the tumor environment and their correlation with tumor progression and susceptibility to metastases. Identification of cells that express genes for immunemodulatory cytokines contributes to our understanding of the molecular basis of biological responses within the microenvironment of primary and metastatic melanomas.

\section{Materials and methods}

\section{Archival Tissues from Melanomas and Melanocytic Nevi}

A total of 45 formalin-fixed, paraffin-embedded tissue specimens were retrieved from the Surgical Pathology archives at UCLA (Los Angeles, CA) and at the Klinikum Nürnberg Nord (Nürnberg, Germany), with Institutional Review Board permission from both institutions. In all, 39 tissue blocks from melanomas and 6 from melanocytic nevi were evaluated: 21 from invasive primary melanomas, 6 from melanomas in situ and 12 from metastatic melanomas (9 lymph nodes (7 sentinel nodes), 1 skin and 2 lung). Original slides from all tissues were reviewed (by AJC and/or PHW) and the diagnosis confirmed. Slides from primary melanomas were assessed for tumor invasiveness, growth pattern and the presence of radial growth phase and vertical growth phase. Primary melanomas showing both radial growth phase and vertical growth phase were categorized as vertical growth-phase melanoma. Clark's level of invasion ${ }^{18}$ and micrometer-measured thickness of invasive primary melanomas ${ }^{19}$ were assessed.

\section{Immunohistochemistry}

Formalin-fixed, paraffin-embedded tissue sections (of $4-\mu \mathrm{m}$ thickness) were deparaffinized in xylene and dehydrated in a series of graded ethanol solutions. Endogenous peroxidase activity was blocked by $3 \%$ hydrogen peroxide for $30 \mathrm{~min}$. Epitope retrieval was performed by boiling in $0.01 \mathrm{M}$ citrate buffer, $\mathrm{pH}$ 6.0, for $30 \mathrm{~min}$. Sections were incubated overnight with a 1:200 polyclonal goat anti-human IL-10 antibody (R\&D Systems, Minneapolis, MN) at $4^{\circ} \mathrm{C}$. The subsequent development of antibody-bridge labeling used the streptavidin-biotin-peroxidase method with biotinylated anti-goat antibodies (Dako, Glostrup, Denmark) and streptavidin horseradish peroxidase (Zymed Laboratories, South San Francisco, CA). Sections were then reacted with the chromogen aminoethyl carbazole (AEC; Zymed Laboratories) and counterstained with hematoxylin and mounted.

\section{One-Step RT In Situ PCR}

\section{Pretreatment}

Formalin-fixed, paraffin-embedded tissue sections were cut at $4 \mu \mathrm{m}$ thickness and placed on silanecoated glass slides, deparaffinized and hydrated through xylene and a graded alcohol series. To determine the optimal duration of protease digestion, slide-mounted tissue sections were digested with trypsin $(0.2$ or $0.4 \mathrm{mg} / \mathrm{ml})$ in phosphatebuffered saline (PBS) for periods that ranged from 30 to $60 \mathrm{~min}$ at $37^{\circ} \mathrm{C}$ (Roche Diagnostics, Mannheim, Germany) and then treated with RNase-free DNase I (Epicentre Biotechnologies, Madison, WI) in PBS at $37^{\circ} \mathrm{C}$ overnight.

\section{Oligonucleotide primer}

The oligonucleotide primer sequences for IL-10 mRNA $^{20}$ are shown as follows: the forward primer, $5^{\prime}$-AGCTCAGCACTGCTCTGTTG-3' ${ }^{\prime}$ and the reverse primer, 5'-GCATTCTTCACCTGCTCCAC- $3^{\prime}$. The RTPCR CDNA product was $428 \mathrm{bp}$.

One-Step RT-PCR

The one-step RT in situ PCR approach used a technique based on descriptions in recent articles. $^{17,21,22}$ The Gene Amp EZ rTth (recombinant Thermus thermophilus) RNA PCR Kit (Applied 
Biosystems, Foster City, CA) was used. Each slide was loaded with the following mixture: $15 \mu \mathrm{l}$ of $5 \times$ EZ rTth buffer, $9 \mu \mathrm{l}$ of $\mathrm{Mn}(\mathrm{OAc})_{2}, 2.4 \mu \mathrm{l}$ each of $\mathrm{dATP} / \mathrm{dCTT} / \mathrm{dGTT} / \mathrm{dTTP}, 3 \mu \mathrm{l}$ of $\mathrm{rTth}$ DNA polymerase (Applied Biosystems), $0.9 \mu \mathrm{l}$ digoxigenin-11dUTP, $2.4 \mu \mathrm{l}$ of $2 \%$ bovine serum albumin (BSA), $1.3 \mu \mathrm{l}$ of RNase inhibitor (Roche Diagnostics), $2.3 \mu \mathrm{l}$ of each primer and $30.7 \mu \mathrm{l}$ diethylpyrocarbonatetreated water. The slides were covered with HybriWell sealing covers (Research Products International, Mount Prospect, IL) and placed on the Gene Amp In Situ PCR System 1000 thermal cycler (Perkin-Elmer, Foster City, CA). cDNA syntheses were performed at $60^{\circ} \mathrm{C}$ for $30 \mathrm{~min}$. After initial denaturation at $94^{\circ} \mathrm{C}$ for $3 \mathrm{~min}$, the cDNA was amplified by 30 cycles of annealing at $55^{\circ} \mathrm{C}$ for $1 \mathrm{~min}$ and denaturation at $94^{\circ} \mathrm{C}$ for $30 \mathrm{~s}$. After RTPCR, the slides were washed in $0.1 \times$ saline-sodium citrate with $2 \% \mathrm{BSA}$ at $60^{\circ} \mathrm{C}$ for $15 \mathrm{~min}$ and rinsed in Tris-buffered saline three times for $5 \mathrm{~min}$.

\section{Immunodetection of RT-PCR products}

After incubation with an alkaline phosphataseconjugated anti-digoxigenin antibody (Roche Diagnostics; 1:200) for $30 \mathrm{~min}$, digoxigenin-labeled RT-PCR products were visualized with nitro blue tetrazolium and 5-bromo-4-chloro-3-indolyl phosphate (Zymed Laboratories) for 3-15 min. The number of immune-reactive cells was estimated semi-quantitatively, as follows: grade $3+$ (high), $>50 \%$ positive cells; grade $2+$ (moderate), $10-50 \%$ positive cells; grade $1+$ (low), $<10 \%$ positive cells; - , negative staining.

\section{Controls}

Negative control preparations were prepared by: (1) omission of the primer pair for IL-10 and (2) omission of the rTth DNA polymerase. Cytocentrifuge slides of Raji human B-lymphoblastic cells, expressing IL-10 ${ }^{23}$ (kindly provided by Dr Jian-Yu Rao, UCLA) served as a positive control.

\section{Statistical Analysis}

Differences in frequencies of positively staining cells in different disease groups were analyzed by Fisher's exact test. Non-parametric data analysis for semi-quantitative scores of IL-10 mRNA reactivity was performed using the Mann-Whitney $U$-test if comparisons involved two groups and the KruskalWallis test if comparisons involved three groups (namely melanoma in situ, invasive primary melanoma and metastatic melanoma). When significance was found, the Kruskal-Wallis test was followed post hoc by the Holm-Bonferroni test for multiple comparisons.

The distribution of cases of IL-10 mRNA-positive expression in melanocytic nevi, melanomas in situ, primary invasive melanomas and metastatic melanomas was evaluated using the Cochran-Armitage trend test. In primary melanomas (melanomas in situ and invasive primary melanomas), correlations between melanoma invasiveness (Clark's level), micrometer-measured tumor thickness (Breslow thickness) and semi-quantitative scores of IL-10 mRNA expression by tumor cells, lymphocytes and macrophages were evaluated by Spearman's rank correlation test. In evaluating Breslow thickness, melanomas in situ (Clark's level 1) were ranked lowest (considered as zero in the micrometer measurement) because the micrometer measurement is only applicable for invasive primary melanomas (Clark's level 2-5). Partial and multiple correlation coefficients were calculated, correlating Clark's level and the reactivity of IL-10 mRNA expression by tumor cells, lymphocytes and macrophages. The model fit was represented by adjusted coefficient of determination $\left(R^{2}\right)$, which determines the reliability of variables to explain variation in the dependent variable (Clark's level).

A multiple logistic regression model was built to predict the vertical growth phase in primary melanomas, using dummy variables for the IL-10 mRNA reactivity data. The model fit was represented by Nagelkerke $R^{2}$ that determines the reliability of variables in the logistic regression model to explain the phenomenon (vertical growth phase).$^{24}$

Statistical significance was considered to be $P<0.05$. Two-tailed $P$-values are quoted throughout. All data were analyzed using the statistical package R: A language and environment for statistical computing (http://www.R-project.org/). ${ }^{25}$

\section{Results}

\section{IL-10 Expression in Tumor Cells}

A positive reaction was indicated by the presence of specific RT-PCR purple- to blue-colored products detectable by microscopic examination. Table 1 summarizes IL-10 mRNA and protein expression by lesional cells of nevi and primary and metastatic melanomas. Melanocytic nevi (0/6) expressed neither IL-10 mRNA (Figure 1a) nor IL-10 protein. IL-10 mRNA was detected in 30 of 39 melanomas $(77 \%)$ and IL-10 protein in 30 of $36(83 \%)$ melanomas. IL-10 mRNA and protein were present in epidermis-associated tumor cells of 2 of 6 melanomas in situ (33\%) (Figure 1b). IL-10 mRNA was detected in 17 of 21 invasive primary melanomas (81\%) (Figure 1c) and IL-10 protein in 17 of 19 invasive primary melanomas (89\%). IL-10 mRNA was present in 11 of 12 metastatic melanomas (92\%) (Figure 1d) and IL-10 protein in 11 of 11 metastatic melanomas (100\%). RT-PCR-amplified IL-10 mRNA and immunohistochemistry-detected protein were located most concentrated in the cytoplasm of tumor cells, although there was minor nuclear staining for mRNA. Fisher's exact test revealed significant differences in IL-10 mRNA and protein expression between melanocytic nevi and primary melanomas 
$(P<0.01)$ and between melanocytic nevi and metastatic melanomas $(P<0.0001)$. IL-10 mRNA and protein were detected at a significantly higher frequency in lesional cells of invasive primary melanomas (Fisher's exact test, $P<0.05$ ) and metastatic melanomas (Fisher's exact test, $P<0.01$ ) relative to melanomas in situ (Table 1). Reactivity scores were recorded in an attempt to quantify the amount of IL-10 mRNA present in melanoma cells at different stages of tumor evolution. These reactivity scores showed statistically significant differences

Table 1 Expression of IL-10 mRNA and protein in tumor cells of primary and metastatic melanomas

\begin{tabular}{|c|c|c|c|c|c|c|}
\hline Tumor & $\begin{array}{r}\text { IL-10 } \\
\text { (RT in } \mathrm{s}\end{array}$ & $\begin{array}{l}\text { mRNA } \\
\text { itu PCR) }\end{array}$ & & $\begin{array}{r}\text { IL-10 P } \\
\text { (immunohist }\end{array}$ & $\begin{array}{l}\text { rotein } \\
\text { chemistry) }\end{array}$ & \\
\hline Melanoma: total & $30 / 39$ & $(77 \%)$ & & $30 / 36$ & $(83 \%)$ & \\
\hline Primary melanoma & $19 / 27$ & $(70 \%)$ & $\square^{\star}$ & $19 / 25$ & $(76 \%)$ & 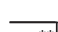 \\
\hline in situ & $2 / 6$ & $(33 \%)$ & $7^{*}$ & $2 / 6$ & $(33 \%)$ & $\overline{7}^{* *}$ \\
\hline invasiviie & $17 / 21$ & $(81 \%)$ & \rfloor & $17 / 19$ & $(89 \%)$ & ] \\
\hline Metastatic melanoma & $11 / 12$ & $(92 \%)$ & $\longrightarrow$ & $11 / 11$ & $(100 \%)$ & - \\
\hline Melanocytic nevi & $0 / 6$ & $(0 \%)$ & $\ldots$ & $0 / 6$ & $(0 \%)$ & \\
\hline
\end{tabular}

${ }^{\star} P<0.05 ;{ }^{* *} P<0.01$.
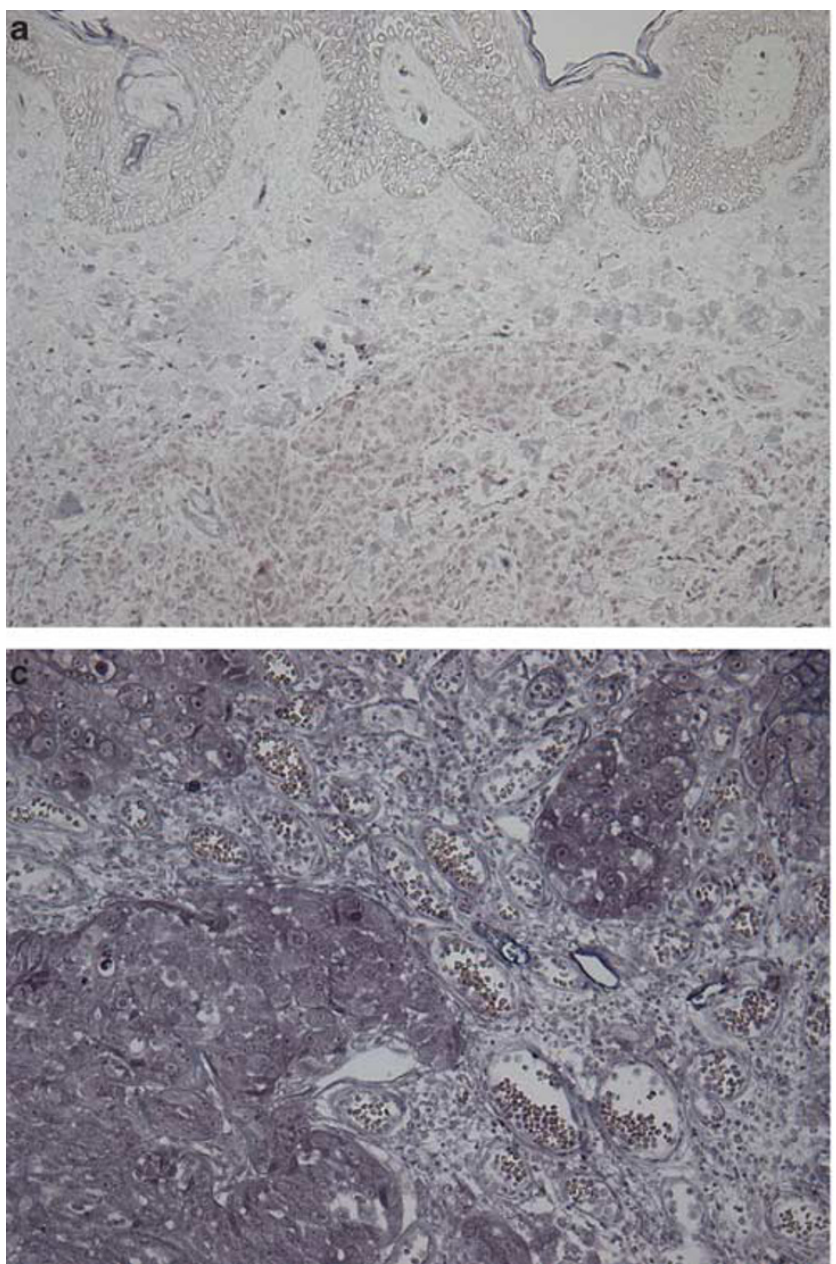

between melanomas in situ, invasive melanomas and metastatic melanomas (Kruskal-Wallis test, $P$ $<0.01$ ). A post hoc Holm-Bonferroni test revealed that IL-10 mRNA reactivity in invasive melanomas and metastatic melanomas was significantly higher than that in melanomas in situ $(P<0.01, P<0.05$, respectively) (Figure 2a).

Neither IL-10 mRNA expression nor IL-10 protein expression was observed in normal melanocytes in the epidermis adjacent to melanomas (data not shown). All negative controls, including omission of the primers and of the Tth DNA polymerase, produced negative results.

\section{IL-10 Expression by Macrophages and Lymphocytes}

IL-10 mRNA (Figure 3a) and protein (Figure 3b) were detected in macrophages and lymphocytes in tissues adjacent to primary melanomas. Many macrophages (including melanophages) located close to a melanoma expressed both IL-10 mRNA
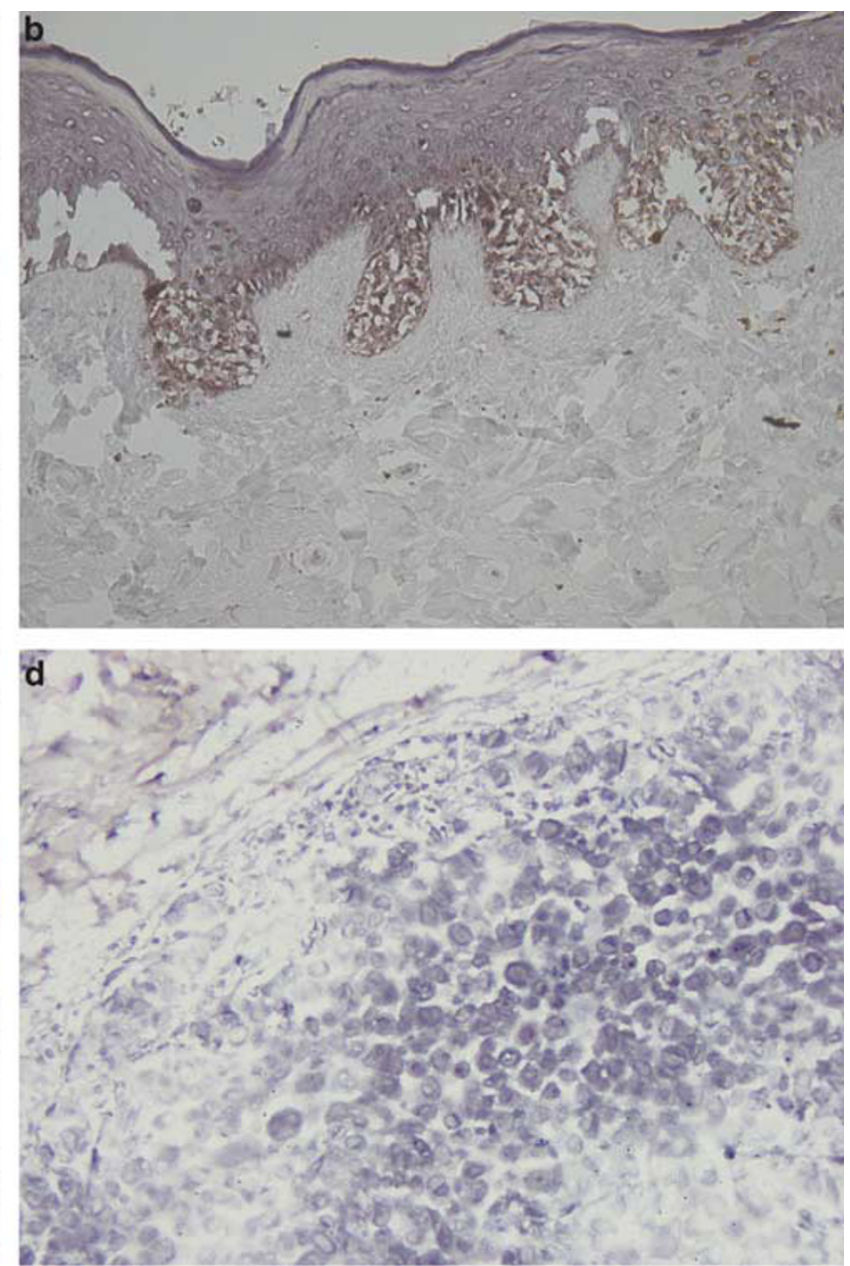

Figure 1 (a) Melanocytic nevus is negative for IL-10 mRNA by the RT in situ PCR technique. (b) Melanoma in situ. The RT in situ PCR technique shows that in some areas, melanoma cells express IL-10. Chromogen: NBT/BCIP. (c) Primary invasive melanoma. The RT in situ PCR technique shows that melanoma cells express IL-10 mRNA. Chromogen: NBT/BCIP. (d) Metastatic melanoma. The RT in situ PCR technique shows that IL-10 mRNA is mostly concentrated in the cytoplasm and that the nucleus contains less reaction product. Chromogen: NBT/BCIP. 

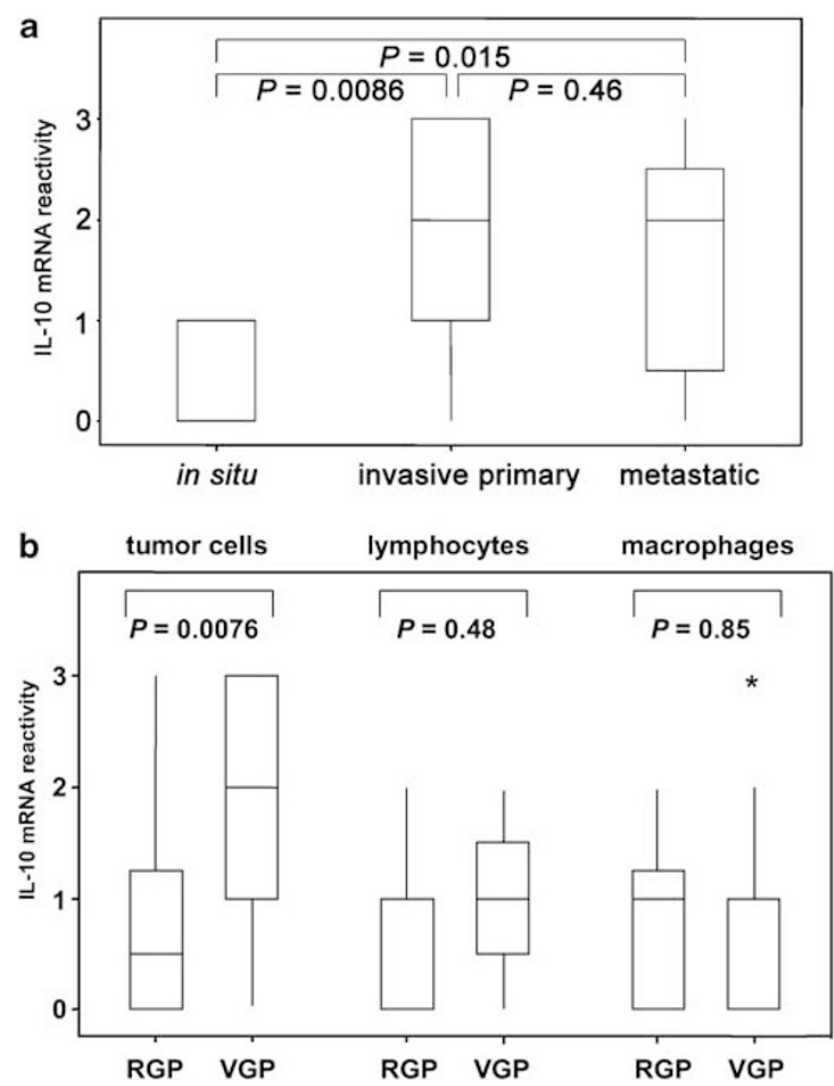

Figure 2 (a) Reactivity scores of IL-10 mRNA expression in melanoma cells. As expressed by the box and whisker plot, the central bar represents the median value, with 25th and 75th percentiles expressed by the box. There is a statistically significant difference in IL-10 between melanoma in situ, invasive melanoma and metastatic melanoma by the Kruskal-Wallis test $(P=0.0057)$. The $\mathrm{IL}-10 \mathrm{mRNA}$ reactivity scores in invasive melanoma and metastatic melanoma are significantly higher than in melanoma in situ $(P=0.0086, P=0.015$, respectively; post hoc Holm-Bonferroni test). (b) Box and whisker plots. IL-10 mRNA expression by melanoma cells is higher in vertical growth-phase melanoma than in radial growth-phase melanoma $(P=0.0076)$. There were no significant differences in IL-10 mRNA reactivity of macrophages or lymphocytes between vertical growth-phase and radial growth-phase melanoma.

and protein, and levels of expression were often high. The IL-10 protein expression by lymphocytes was more limited, occurring in a few lymphocytes at less intense levels than observed in macrophages. The IL-10 mRNA expression by macrophages and lymphocytes was similarly frequent in infiltrates adjacent to vertical and radial growth-phase melanomas (Figure 2b).

In a melanoma metastatic to the lung, IL-10 mRNA expression was observed in melanoma cells (Figure 3c and d) and tumor-adjacent alveolar macrophages (Figure 3e).

\section{Correlation between Tumor Invasiveness and IL-10 mRNA Expression}

The Cochran-Armitage trend test showed that IL-10 mRNA reactivity, absent in melanocytic nevi, occurred at progressively increasing frequency in melanomas in situ, invasive primary melanomas and metastatic melanomas $(P<0.0001)$. In 27 primary melanomas, including melanomas in situ and invasive melanomas, Spearman's correlation analysis (Table 2) showed a very significant direct correlation between IL-10 mRNA expression by tumor cells and increasing Clark's level of invasion $(r=0.53)$ and increasing Breslow thickness $(r=0.41)$. There was a significant correlation between the expression of IL-10 mRNA in tumoradjacent lymphocytes and the expression in tumoradjacent macrophages $(r=0.47)$.

In a model in which Clark's level was a dependent variable, the multiple correlation coefficient $(R)$ was 0.68 , and the adjusted $R^{2}$ was 0.40 . By removing the effects of the reactivity of macrophages and lymphocytes, the partial correlation coefficient between Clark's level and the reactivity of tumor cells was 0.62 , slightly higher than the corresponding Spearman's correlation coefficient $(r=0.53)$. By removing the effects of Clark's level and the reactivity of tumor cells, the partial correlation coefficient between reactivity of lymphocytes and macrophages was 0.52. The partial correlation coefficients between the reactivity of macrophages and tumor cells or Clark's level were significant, whereas the corresponding Spearman's correlation coefficients were not significant. By removing the effects of reactivity of tumor cells and of lymphocytes, the reactivity of macrophages inversely correlated with Clark's level $(r=-0.49)$. By removing the effects of Clark's level and reactivity of lymphocytes, the reactivity of tumor cells correlated with that of macrophages $(r=0.51)$.

When we compared IL-10 mRNA reactivity scores of radial growth-phase and vertical growth-phase melanomas, scores were significantly higher in vertical growth-phase melanomas $(P<0.01)$ (Figure $2 b)$. To predict that a melanoma is in the vertical growth phase, a multiple logistic regression model was built using dummy variables for the expression of IL-10 mRNA (Table 3). Nagelkerke $R^{2}$ for the logistic regression model was $0.52(P<0.05)$, indicating a good fit of the data to the model. In this model, moderate-to-high IL-10 mRNA expression by tumor cells was confirmed to be significantly associated with the vertical growth phase of melanoma $(P<0.05)$.

\section{Discussion}

Melanomas evolve through sequential steps as the capacities for local invasion and metastatic competence evolve. During the radial growth phase, melanoma cells proliferate within the epidermis with, at most, limited non-tumorigenic penetration of the dermis by single cells or very small groups of cells (invasive radial growth phase) in the later stages of radial growth-phase evolution. ${ }^{26,27}$ Radial 

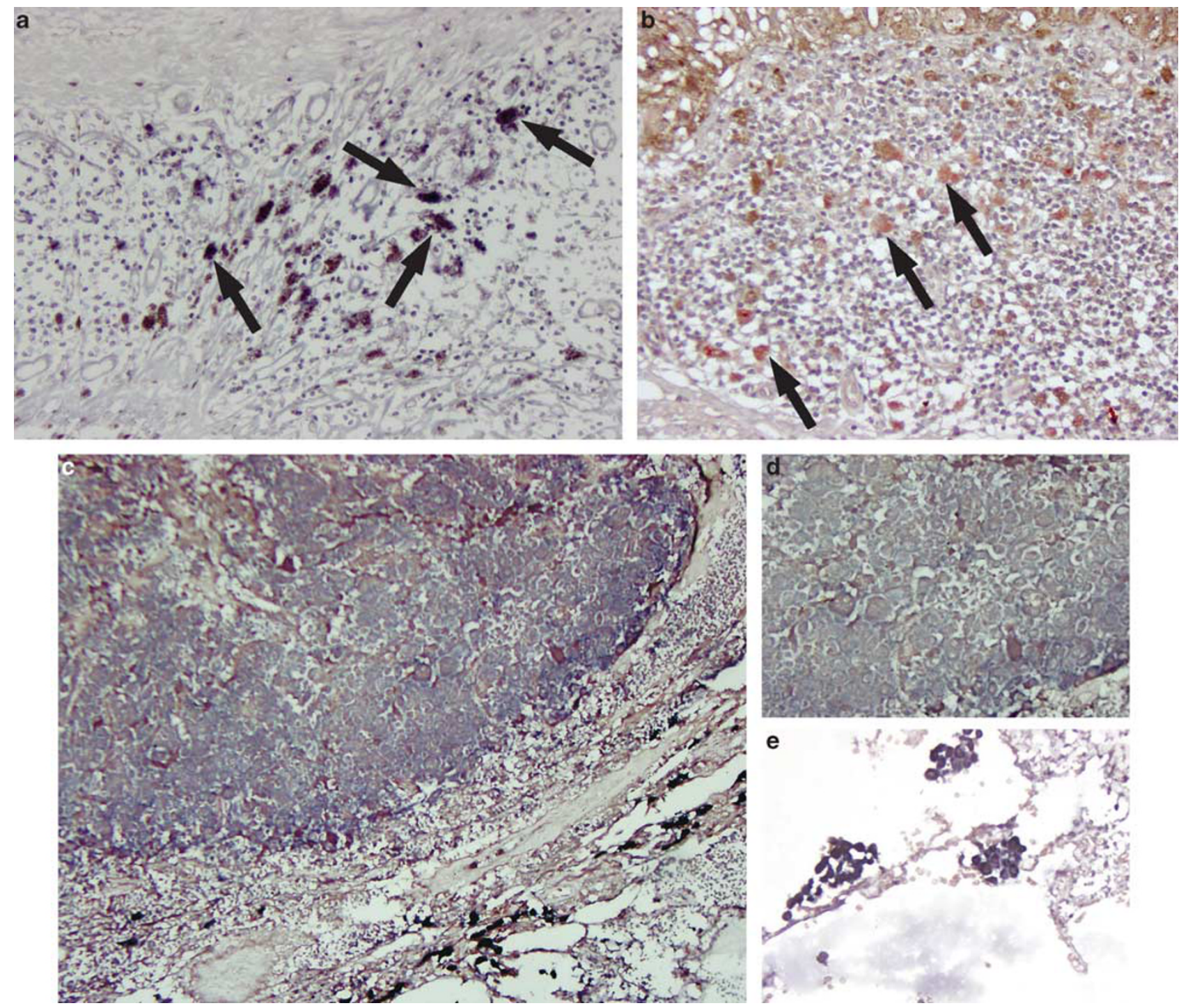

Figure 3 (a) IL-10 mRNA expression in lymphocytes and macrophages in primary melanoma. Macrophages including melanophages are strongly positive for IL-10 (dark blue color, marked by arrows). IL-10-positive lymphocytes are also present (blue color). The brown color is melanin. (b) Immunohistochemistry reveals that IL-10 is strongly expressed in some of the macrophages including melanophages (red color, marked by arrows) and in scattered lymphocytes (red color, marked by asterisk). (c, d) In metastatic melanoma of the lung, IL-10 mRNA is diffusely expressed in tumor cells (blue color). (e) Alveolar macrophages near the metastatic foci also express IL-10 mRNA in metastatic melanoma of the lung (blue color).

Table 2 IL-10 mRNA reactivity in tumor cells, macrophages and lymphocytes correlated with the stage of melanoma evolution (melanoma in situ and invasive melanoma)

\begin{tabular}{lcccc}
\hline & Clark's level & Melanoma cells & Macrophages & Lymphocytes \\
\hline Breslow thickness & 1 & $\mathbf{0 . 4 1}^{*}$ & -0.07 & 0.27 \\
Clark's level & 1 & $\mathbf{0 . 5 3}^{* *}$ & -0.10 & 0.37 \\
Melanoma cells & $\mathbf{0 . 6 2}^{* *}$ & 1 & 1 & 0.23 \\
Macrophages & $-\mathbf{0 . 4 9}^{*}$ & $\mathbf{0 . 5 1}^{*}$ & $\mathbf{0 . 5 2}^{* *}$ & $\mathbf{0 . 4 6}^{*}$ \\
Lymphocytes & 0.38 & -0.19 & 1 \\
\hline
\end{tabular}

$R=0.68$ (dependent variable: Clark's level, $P=0.0016$ ). Adjusted $R^{2}=0.40$. Melanoma in situ was ranked lowest in consideration of Breslow thickness.

Bold values represent significant correlations $\left({ }^{*} P<0.05,{ }^{*} P<0.01\right)$

Above the main diagonal: Spearman's correlation coefficients and $P$-values.

Below the main diagonal: partial correlation coefficients and $P$-values. 
Table 3 Multiple logistic regression model to predict characteristics of the vertical growth phase of melanoma $(P=0.024$, Nagelkerke $R^{2}=0.52$ )

\begin{tabular}{|c|c|c|c|}
\hline Variables & Coefficient & s.e. & $\mathrm{P}$-value \\
\hline Constant & -1.46 & 1.17 & 0.21 \\
\hline Low IL-10 mRNA expression by tumor cells & 1.96 & 1.59 & 0.22 \\
\hline Moderate-to-high IL-10 mRNA expression by tumor cells & 4.40 & 1.72 & $0.011^{*}$ \\
\hline Low-to-moderate IL-10 mRNA expression by lymphocytes & 1.46 & 1.81 & 0.42 \\
\hline Low IL-10 mRNA expression by macrophages & -1.96 & 1.79 & 0.27 \\
\hline Moderate-to-high IL-10 mRNA expression by macrophages & -3.89 & 2.39 & 0.10 \\
\hline
\end{tabular}

growth-phase melanoma treated by complete surgical excision has a very low risk of local regrowth or metastasis. When melanoma cells acquire the capacity to form a tumor (tumorigenicity) and infiltrate more extensively into the dermis and subcutaneous tissues, the melanoma is considered as being in the vertical growth phase and having acquired the capacity to metastasize. ${ }^{27-29}$ In line with a recent report, ${ }^{30}$ our results indicate that IL-10 production by melanoma cells increases as melanomas progress through these evolutionary steps, paralleling the development of metastatic competence. This alteration in the capacity to generate and secrete IL-10 is associated with increasing prognostic implications of transition from the radial to the vertical growth phase and the developing risk of regional and visceral metastases. In primary melanomas, the presence of detectable IL-10 mRNA in tumor cells correlated with increasing Clark's level, an index of progression of the vertical growth phase to involvement of the deeper dermis and subcutis and a direct correlate of increasing metastatic potential. The frequency of melanoma cells with detectable IL-10 mRNA also increased with increasing Breslow thickness, but the correlation was less strong than with Clark's level. These data indicate the frequency of melanoma cells that express detectable IL-10 and do not indicate the actual amounts of IL-10 expressed by tumor cells.

The mechanisms that drive IL-10 overexpression in melanoma cells are not fully known. Overexpression of IL-10 may be due to chromosomal aberrations that are known to occur in melanomas. A gain of chromosome 1q (on which the human IL10 gene is cytogenetically located) has been reported in melanomas ${ }^{31-33}$ and could induce IL-10 overexpression.

Melanoma-derived IL-10 may suppress the differentiation and function of dendritic cells, ${ }^{10}$ and levels of IL-10 are reportedly higher in sentinel nodes than in non-sentinel nodes in patients with residual primary melanoma or metastatic melanoma in the sentinel node. ${ }^{34,35}$ Thus, the suppression of immunological functions in the melanoma-draining sentinel node may be downregulated by immunosuppressive cytokines derived from melanoma (and other) cells located outside the lymph node. IL-10 produced by melanoma cells, acting as an autocrine growth factor, can auto-stimulate proliferation of cells that produce it leading to even higher levels of IL-10. ${ }^{36}$ In this study, IL-10 expression by melanoma cells correlated with the extent of primary tumor invasion. The expression of IL-10 mRNA was moderate to high in tumor cells of vertical growthphase melanoma and metastatic melanoma. This suggests that IL-10 production by melanoma cells may be related to the primary tumor's capacity to proliferate, expand and invade adjacent tissues and acquire critical competence for metastasis. IL-10 expression was more significantly correlated with increasing invasion of the deeper layers of the skin (Clark's level), than with micrometer-measured tumor thickness (Breslow). This suggests that once the cellular characteristics of the vertical growthphase are established, increasing tumor cell numbers, as evidenced by increasing Breslow thickness (a surrogate for tumor volume), does not change the relative frequency of IL-10-expressing tumor cells. It is also noteworthy that individual tumor cells of some melanomas in situ may produce IL-10, suggesting that even before transgression of the basement membrane, melanoma cells may generate releasable molecules capable of inducing immune suppression. This is in line with the view that melanomas can modulate the local microenvironment and that such modulation is part of the mechanisms that facilitate tumor growth and invasion.

Melanocytic nevi are benign, focal proliferations of melanocyte-derived cells that can remain stable for many years, but may infrequently act as precursors of melanoma. In this study, IL-10 expression was absent from nevus cells and normal melanocytes in sections of ostensibly normal skin adjacent to nevi and melanomas. IL-10 can be detected in cultured melanocytes in vitro, but the level of IL-10 expression in such cultures is extremely low compared with that in melanomas. ${ }^{37}$

Peritumoral inflammatory cells have a key role in the promotion or subversion of adaptive immunity and in inflammatory circuits that may promote tumor growth and progression. ${ }^{38-40}$ Tumor-associated macrophages have dual roles in relation to neoplasms. Although tumor-associated macrophages can kill neoplastic cells, following activation by IL-2, interferon and IL-12, tumorassociated macrophages 'educated' by the tumor 
microenvironment can produce potent angiogenic and lymphangiogenic growth factors, as well as cytokines and protease mediators that can promote tumor progression and metastasis. ${ }^{12,39,41,42}$ This study shows that IL-10 is produced by tumor-associated macrophages and tumor-related lymphocytes associated with early stages of tumor evolution, including melanoma in situ. Macrophages associated with thin primary melanomas contained more IL-10 mRNA than did those associated with deeper and thicker primary melanomas, suggesting that peak generation of IL-10 by tumor-associated macrophages may be selectively associated with the earliest phases of tumor invasion. The presence of detectable IL-10 mRNA in macrophages correlated directly with detectable IL-10 mRNA in melanoma cells, making it likely that both types of cells contribute to IL-10induced immune suppression. We also demonstrated IL-10 production by alveolar macrophages located close to metastatic melanoma in the lung. Such macrophages located close to a metastasis along with the actual metastatic tumor cells could also induce local immune suppression. However, we cannot entirely exclude the possibility that the highly sensitive RT in situ PCR technique may augment acquired nucleotides in the cytoplasm of macrophages, which have phagocytosed melanoma cell components (including IL-10 mRNA and protein).

Immunoregulatory T cells, CD4 + CD25 + T-regulatory cells are capable of suppressing anti-tumor immunity, ${ }^{30,43}$ and this class of cells derived from lymph nodes that contain metastatic melanoma can produce IL-10 upon allogeneic stimulation, but Th cells have also been reported to secrete IL-10. ${ }^{44}$ Immunosuppressive monocytes/macrophages having the same characteristics as tumor-associated macrophages can be induced by the influence of T-regulatory cells. ${ }^{45}$ In this study, the presence of detectable IL-10 mRNA in macrophages correlated directly with detectable IL-10 mRNA in lymphocytes. Further investigation will investigate whether these IL-10-secreting peritumoral lymphocytes are T-regulatory cells. Thus, through elaboration of immunosuppressive cytokines such as IL-10, macrophages and lymphocytes contribute to the immune suppression that impedes the development of effective anti-tumor immune responses. They may induce localized and specific immune paralysis at both primary and metastatic sites, facilitating growth of primary and metastatic melanomas. Further studies are required to elucidate the specific mechanisms of cross-talk between different cell types within the tumor and surrounding inflammatory cells.

In summary, IL-10 production by tumor cells increases as primary melanomas progress with important prognostic implications for the transition from melanoma in situ to invasive melanoma and metastatic melanoma. Macrophages and lymphocytes infiltrating around primary and metastatic melanomas may also contribute to immune suppression. The release of immunosuppressive factors from melanoma cells, tumor-associated macrophages and lymphocytes seem to contribute to localized and specific immune downregulation at both primary and metastatic sites. The findings are consistent with the proposal that IL-10, acting with other cytokines, induces immune downregulation that may render sentinel nodes susceptible to the development of metastases. Understanding the interaction between tumor cells and the immune network in the immune suppression of anti-tumor immunity will help to provide insights that will permit the introduction of future novel therapeutic approaches to the management of patients with melanoma.

\section{Acknowledgement}

This study was supported by the NIH/NCI grant P01 CA 29605 (Administered by the John Wayne Cancer Institute, Saint John's Health Center, Santa Monica, CA).

\section{Disclosure/conflict of interest}

Dr Cochran acts as a consultant to the John Wayne Cancer Institute.

\section{References}

1 Cochran AJ, Pihl E, Wen DR, et al. Zoned immune suppression of lymph nodes draining malignant melanoma: histologic and immunohistologic studies. J Natl Cancer Inst 1987;78:399-405.

2 Lana AMA, Wen DR, Cochran AJ. The morphology, immunophenotype and distribution of paracortical dendritic leucocytes in lymph nodes regional to cutaneous melanoma. Melanoma Res 2001;11:401-410.

3 Cochran AJ, Morton DL, Stern S, et al. Sentinel lymph nodes show profound downregulation of antigenpresenting cells of the paracortex: implications for tumor biology and treatment. Mod Pathol 2001;14: 604-608.

4 Cochran AJ, Huang RR, Lee J, et al. Tumour immunology-tumour-induced immune modulation of sentinel lymph nodes. Nat Rev Immunol 2006;6:659-670.

5 Dummer W, Becker JC, Schwaaf A, et al. Elevated serum levels of interleukin-10 in patients with metastatic malignant melanoma. Melanoma Res 1995;5: 67-68.

6 Dummer W, Bastian BC, Ernst N, et al. Interleukin-10 production in malignant melanoma: preferential detection of IL-10-secreting tumor cells in metastatic lesions. Int J Cancer 1996;66:607-610.

7 Boyano MD, Garcia-Vazquez MD, Lopez-Michelena T, et al. Soluble interleukin-2 receptor, intercellular adhesion molecule-1 and interleukin-10 serum levels in patients with melanoma. Br J Cancer 2000;83: 847-852.

8 Chen Q, Daniel V, Maher DW, et al. Production of IL-10 by melanoma cells: examination of its role in immunosuppression mediated by melanoma. Int J Cancer 1994;56:755-760. 
9 Krüger-Krasagakes S, Krasagakis K, Garbe C, et al. Expression of interleukin 10 in human melanoma. Br J Cancer 1994;70:1182-1185.

10 Gerlini G, Tun-Kyi A, Dudli C, et al. Metastatic melanoma secreted IL-10 down-regulates CD1 molecules on dendritic cells in metastatic tumor lesions. Am J Pathol 2004;165:1853-1863.

11 Biggs MW, Eiselein JE. Suppression of immune surveillance in melanoma. Med Hypotheses 2001; 56:648-652.

12 Pollard JW. Tumour-educated macrophages promote tumour progression and metastasis. Nat Rev Cancer 2004;4:71-78.

13 O'Connell J. RT-PCR-based approaches to generate probes for mRNA detection by in situ hybridization. In: O’Connell J (ed). Methods in Molecular Biology: RT-PCR Protocols. Humana Press: Totowa, NJ, 2002. pp 213-224.

$14 \mathrm{Li}$ PX, Cheng L, Wen DR, et al. Demonstration of cytoplasmic tyrosinase mRNA in tissue-cultured cells by reverse transcription (RT) in situ polymerase chain reaction (PCR) and RT PCR in situ hybridization. Diagn Mol Pathol 1997;6:26-33.

15 Guo J, Wen DR, Huang RR, et al. Detection of multiple melanoma-associated markers in melanoma cell lines by RT in situ PCR. Exp Mol Pathol 2003;74:140-147.

16 Guo J, Cheng L, Wen DR, et al. Detection of tyrosinase mRNA in formalin-fixed, paraffin-embedded archival sections of melanoma, using the reverse transcriptase in situ polymerase chain reaction. Diagn Mol Pathol 1998;7:10-15.

17 Itakura E, Huang RR, Wen DR, et al. RT in situ PCR detection of MART-1 and TRP-2 mRNA in formalinfixed, paraffin-embedded tissues of melanoma and nevi. Mod Pathol 2008;21:326-333.

18 Clark Jr WH, From L, Bernardino EA, et al. The histogenesis and biologic behavior of primary human malignant melanomas of the skin. Cancer Res 1969;29:705-727.

19 Breslow A. Thickness, cross-sectional areas and depth of invasion in the prognosis of cutaneous melanoma. Ann Surg 1970;172:902-908.

20 Colgan SP, Hershberg RM, Furuta GT, et al. Ligation of intestinal epithelial CD1d induces bioactive IL-10: critical role of the cytoplasmic tail in autocrine signaling. Proc Natl Acad Sci USA 1999;96: 13938-13943.

21 Nuovo GJ. Co-labeling using in situ PCR: a review. J Histochem Cytochem 2001;49:1329-1339.

22 Adam PJ, Boyd R, Tyson KL, et al. Comprehensive proteomic analysis of breast cancer cell membranes reveals unique proteins with potential roles in clinical cancer. J Biol Chem 2003;278:6482-6489.

23 Benjamin D, Knobloch TJ, Dayton MA. Human B-cell interleukin-10: B-cell lines derived from patients with acquired immunodeficiency syndrome and Burkitt's lymphoma constitutively secrete large quantities of interleukin-10. Blood 1992;80:1289-1298.

24 Nagelkerke NJD. A note on a general definition of the coefficient of determination. Biometrika 1991;78: 691-692.

25 R Development Core Team. R: A Language and Environment for Statistical Computing. R Foundation for Statistical Computing: Vienna, 2010.

26 Herlyn M, Clark WH, Rodeck U, et al. Biology of tumor progression in human melanocytes. Lab Invest 1987;56:461-474.
27 Elder DE, Rodeck U, Thurin J, et al. Antigenic profile of tumor progression stages in human melanocytic nevi and melanomas. Cancer Res 1989;49: 5091-5096.

28 Clark Jr WH, Elder DE, Van Horn M. The biologic forms of malignant melanoma. Hum Pathol 1986;17:443-450.

29 Nyormoi O, Bar-Eli M. Transcriptional regulation of metastasis-related genes in human melanoma. Clin Exp Metastasis 2003;20:251-263.

30 Polak ME, Borthwick NJ, Gabriel FG, et al. Mechanisms of local immunosuppression in cutaneous melanoma. Br J Cancer 2007;96:1879-1887.

31 Bastian BC, LeBoit PE, Hamm H, et al. Chromosomal gains and losses in primary cutaneous melanomas detected by comparative genomic hybridization. Cancer Res 1998;58:2170-2175.

32 Balazs M, Adam Z, Treszl A, et al. Chromosomal imbalances in primary and metastatic melanomas revealed by comparative genomic hybridization. Cytometry 2001;46:222-232.

33 Sargent LM, Nelson MA, Lowry DT, et al. Detection of three novel translocations and specific common chromosomal break sites in malignant melanoma by spectral karyotyping. Genes Chromosomes Cancer 2001;32:18-25.

34 Lee JH, Torisu-Itakara H, Cochran AJ, et al. Quantitative analysis of melanoma-induced cytokine-mediated immunosuppression in melanoma sentinel nodes. Clin Cancer Res 2005;11:107-112.

35 Torisu-Itakura H, Lee JH, Scheri RP, et al. Molecular characterization of inflammatory genes in sentinel and nonsentinel nodes in melanoma. Clin Cancer Res 2007;13:3125-3132.

36 Lazar-Molnar E, Hegyesi H, Toth S, et al. Autocrine and paracrine regulation by cytokines and growth factors in melanoma. Cytokine 2000;12:547-554.

37 Brewer G, Saccani S, Sarkar S, et al. Increased interleukin-10 mRNA stability in melanoma cells is associated with decreased levels of $\mathrm{A}+\mathrm{U}$-rich element binding factor AUF1. J Interferon Cytokine Res 2003; 23:553-564.

38 Balkwill F, Mantovani A. Inflammation and cancer: back to Virchow? Lancet 2001;357:539-545.

39 Coussens LM, Werb Z. Inflammation and cancer. Nature 2002;420:860-867.

40 Brigati C, Noonan DM, Albini A, et al. Tumors and inflammatory infiltrates: friends or foes? Clin Exp Metastasis 2002;19:247-258.

41 Torisu H, Ono M, Kiryu H, et al. Macrophage infiltration correlates with tumor stage and angiogenesis in human malignant melanoma: possible involvement of TNFalpha and IL-1alpha. Int J Cancer 2000; 85:182-188.

42 Ilkovitch D, Lopez DM. Immune modulation by melanoma-derived factors. Exp Dermatol 2008;17: 977-985.

43 Terabe M, Berzofsky JA. Immunoregulatory $\mathrm{T}$ cells in tumor immunity. Curr Opin Immunol 2004;16:157-162.

44 Viguier M, Lemaitre F, Verola O, et al. Foxp3 expressing CD4+CD25(high) regulatory $\mathrm{T}$ cells are overrepresented in human metastatic melanoma lymph nodes and inhibit the function of infiltrating T cells. J Immunol 2004;173:1444-1453.

45 Tiemessen MM, Jagger AL, Evans HG, et al. $\mathrm{CD}^{+}{ }^{+} \mathrm{CD} 25^{+} \mathrm{Foxp}^{+}$regulatory $\mathrm{T}$ cells induce alternative activation of human monocytes/macrophages. Proc Natl Acad Sci USA 2007;104:19446-19451. 